LIVER DISEASE

\title{
Identification of expandable human hepatic progenitors which differentiate into mature hepatic cells in vivo
}

\author{
G Nowak, B-G Ericzon, S Nava, M Jaksch, M Westgren, S Sumitran-Holgersson
}

See end of article for authors' affiliations

Correspondence to:

Dr S Sumitran-Holgersson, Department of

Transplantation Surgery,

B56, Huddinge University

Hospital, S-14186

Stockholm, Sweden

suchitra.holgersson@

cfss.ki.se

Revised version received

14 February 2005

Accepted for publication

7 March 2005
Gut 2005;54:972-979. doi: 10.1136/gut.2005.064477

Background: Liver diseases include a wide spectrum of both acute and chronic conditions which are associated with significant morbidity and mortality worldwide. Hepatocyte transplantation has therapeutic potential in the treatment of liver diseases, but its clinical use is hampered by the lack of donor tissue. Generation of hepatocytes in vitro from adult or fetal liver cell progenitors or, alternatively, identification of a progenitor population which in vivo can generate mature liver cells could solve this problem.

Methods: CD117+/CD34+/Lin - human fetal liver cells were isolated by magnetic cell sorting and expanded in culture. Both freshly isolated and in vitro expanded cells in various passages were studied for their ability to be functional in hepatic parenchyma following D-galactosamine (GalN) induced injury in nude C57 black mice.

Results: Freshly isolated and in vitro expanded CD117+/CD34+/Lin- cells, when transplanted intrasplenically into GalN treated mice, morphologically and functionally differentiated into hepatocytes and cholangiocytes. Human specific albumin, $\alpha$ fetoprotein, cytokeratin 19, and antitrypsin mRNA were expressed in mouse liver. In addition, the human progenitor cells expressed glucose-6-phosphatase, glycogen, albumin, gamma glutamyl transpeptidase, and dipeptidyl peptidase IV after transplantation. Expanded cells in various passages maintained their capacity to differentiate into functional liver cells. Conclusions: Fetal liver CD117+/CD34+/Lin - progenitors and their progeny proliferated in vitro and also functionally differentiated into mature hepatic cells in an acute liver injury model. Successful in vitro expansion of liver progenitor cells provides a basis for developing cell therapy strategies, metabolic and toxicity testing systems, and may serve as a vehicle for gene therapy.
O rthotopic liver transplantation is the only available therapy for patients with end stage liver failure. Unfortunately, the availability of donor organs is limited and many patients die each year waiting for liver transplants. Recently, transplantation of healthy hepatocytes into a diseased liver has been used as an alternative therapy. ${ }^{2-4}$ However, once again, shortage of organ donors has limited the clinical application of hepatocyte cell transplantation. If donor cells are to replace sufficient liver mass to restore the life sustaining function of the liver, the cells must be able to expand in vitro or increase in number by cell replication after transplantation. Thus generation of hepatocytes in vitro from adult or fetal liver cell progenitors or, alternatively, identification of a progenitor population which in vivo can proliferate and generate mature liver cells may be a successful strategy.

The ability to repopulate the liver with transplanted stem/ progenitor cells has raised hopes for improving outcome in acute liver failure..$^{5-11}$ Tissue residing stem cells of the liver have been identified in adult rodent and human livers by coexpression of haematopoietic stem cell markers (for example, CD34, Thy-l, and c-kit) and hepatocytic lineage markers (cytokeratin (CK)-18, CK19, $\alpha$ fetoprotein, and albumin). ${ }^{12}{ }^{13}$ Although stem/progenitor cells from adult organs may generate functional liver cells, such cells are rare. Achievement of positive outcomes in many clinical protocols involving liver progenitor cells has been handicapped by the limited numbers of liver repopulating cells available to actually bring about therapy. The mammalian fetal liver contains a compartment of colony forming cells with high proliferative potential, consisting of primitive haematopoietic progenitors and, most likely, pluripotent stem cells. ${ }^{14}$ The fetal liver is therefore an attractive source for isolation of hepatic progenitors for transplantation. In our previous observations, we found that the first trimester human fetal liver (gestational weeks $5-10$ ) is rich in cells expressing haematopoietic markers such as CD117 and CD34, but no or few cells expressing hepatic markers such as albumin and CK19. Cells expressing hepatic markers appeared first in the second trimester. We therefore isolated CD117+/CD34+ cells from gestational weeks 7-9 and tested their ability to differentiate into cells that express hepatic markers. Our preliminary results indicated that in vitro, these cells seemingly differentiated into cells expressing albumin and CK19 (submitted). Therefore, in the present study the main aim was to isolate CD117+/CD34+ "putative hepatic progenitors" from human fetal livers and further characterise them with regard to their proliferative and functional capacity to differentiate into mature hepatic cells.

\section{MATERIALS AND METHODS}

Isolation of human fetal liver cells

Permission for the study was granted from the local ethics committee at Huddinge University Hospital. Human fetal liver (FL) tissues were obtained from aborted fetuses at 610 weeks' gestation in accordance with Swedish guidelines. A modified vacuum curettage was performed..$^{15}$ Gestational age was estimated according to specific anatomical markers and is given as menstrual age. ${ }^{16}$ The abortions were performed in pregnancies with no apparent abnormalities,

Abbreviations: FL, fetal liver; PBS, phosphate buffered saline; DMEM, Dulbecco's modified Eagle medium; HGF, hepatocyte growth factor; EGF, epidermal growth factor; bFGF, basic fibroblast growth factor; $\mathrm{CM}$, conditioned medium; DAB-Ni, diaminobenzidine tetrahydrochloride-nickel; GalN, D-galactosamine; GGT, gamma glutamyl transpeptidase; G-6-P, glucose-6-phosphatase; G-6-PD, glucose-6phosphate dehydrogenase; RT-PCR, reverse transcriptase-polymerase chain reaction; $C K$, cytokeratin; $H C V$, hepatitis $C$ virus 
and no fetuses with anomalies were included. FL was dissected and placed in a sterile tube containing RPMI 1640 medium (Gibco, Invitrogen Corp., UK). The livers were then disintegrated into a single cell suspension by passage through a $70 \mu \mathrm{m}$ metal mesh. The single cell suspension was centrifuged at $200 \mathrm{~g}$ for 10 minutes to pellet the cells. All women donating fetal tissue had been serologically screened for syphilis, toxoplasmosis, rubella, human immunodeficiency virus 1 , cytomegalovirus, hepatitis B and C, parovirus, and herpes simplex types 1 and 2 .

\section{Isolation and phenotyping of human primitive progenitors from fetal livers cells}

Single cell suspensions were prepared from each FL in gestational weeks 6-10. Cells were isolated using the human primitive progenitor cell enrichment isolation kit (Stem Cell Technologies, Vancouver, Canada) followed by the magnetic activated cell separation magnetic bead system (Stem Cell Technologies), as described by the manufacturers. The method is based on negative selection of this population using a depletion cocktail, including antibodies to 12 lineage specific cell surface antigens (anti-CD2, -CD3, -CD14, -CD16, -CD19, -CD24,-CD36, -CD38, -CD45RA, -CD56, -CD66b, and -glycophorin A). On every occasion, the recovered progenitor cells were immediately analysed by the flow cytometer to ensure that no contaminating Lin+ cells were present. Flow cytometry was performed using the following antihuman antibodies: FITC conjugated anti-CDI17, PE conjugated antiCD34, anti-CD90, anti-CD45 (Becton Dickinson, USA), an FITC conjugated goat antibody against human albumin (Natutec, Frankfurt, Germany), a non-conjugated antihuman CK19 (Neomarker, USA, and Pharmingen, USA), and secondary subclass specific antibodies goat antimouse IgGl (FITC/Texas red) and goat antimouse IgG2a (FITC/Texas red) (Jackson Immunoresearch, USA). For CK19 detection, intracellular staining was performed after cell permeabilisation using 5\% saponin in phosphate buffered saline (PBS), as described previously. ${ }^{17}$

Our previous analysis of 94 human fetal livers in gestational weeks 5-18 using various hepatic markers such as epithelial cell adhesion molecule, hepatocyte growth factor receptor (c-Met), albumin, and CK19 showed that one of the first surface markers expressed by hepatic cells in the human developing liver is c-Met (submitted). To test which specific populations express c-Met, three colour staining was performed. Cells were first stained with an unconjugated anti-c-Met (Research Diagnostics, Germany) antibody (isotype IgG2a) for 30 minutes at $4^{\circ} \mathrm{C}$, washed, and intracellular staining for CK19 (isotype IgG1) was performed after cell permeabilisation using 5\% saponin in PBS. After washing, cells were stained with the FITC conjugated antihuman albumin antibody, a secondary subclass specific goat antimouse IgGl (PE conjugated), and goat antimouse IgG2a (PerCP conjugated; Jackson Immunoresearch). For all flow cytometric analysis, corresponding control isotypes were used for evaluation of non-specific binding of monoclonal antibodies. The cells were analysed on a Becton Dickinson flow cytometer (FACSorter). FACS analysis demonstrated that the phenotype of the human primitive progenitor cells isolated from FLs was CD117+/CD34+/Lin-.

\section{In vitro cultivation and detection of proliferation of CD $117+/$ CD34+/Lin - cells}

The recovered progenitor cells were tested for viability, after which cells were seeded in plastic Petri dishes coated with collagen type I (Biocoat; Becton and Dickinson, New Jersey, USA) and cultivated in Dulbecco's modified Eagle medium (DMEM) (Gibco, Invitrogen, Stockholm, Sweden) containing $10 \%$ inactivated fetal calf serum, penicillin and streptomycin,
5\% L-glutamine, 5\% minimum essential amino acids, $50 \mathrm{ng} / \mathrm{ml}$ hepatocyte growth factor (HGF) (R\&D Systems, Abingdon, UK), $20 \mathrm{ng} / \mathrm{ml}$ epidermal growth factor (EGF) (R\&D Systems), and $10 \mathrm{ng} / \mathrm{ml}$ basic fibroblast growth factor (bFGF) (R\&D Systems). Every third day the medium was collected, centrifuged, sterile filtered, and used as conditioned medium (CM). All subsequent subculturing was performed using $20 \% \mathrm{CM}$.

For identification of cells with proliferative capacity, the following procedure was used. Cells in passages 4, 6, and 12 were allowed to grow on collagen I coated glass slides and incubated with the thymidine analogue BrdU $(30 \mathrm{mM})$ for 30 minutes, washed, and fixed using 30\% acetone in methanol for one minute. After two washes with PBS, and blocking with $1 \%$ bovine serum albumin in PBS for one hour at room temperature, cells were incubated with anti-CK19, diluted 1:100 (in PBS) at $4^{\circ} \mathrm{C}$ for two hours and then stained with a Texas red conjugated goat antimouse secondary antibody, diluted 1:500 (in PBS), and an FITC conjugated antihuman albumin antibody (1:100). After incubation for one hour at $4^{\circ} \mathrm{C}$ and washing, cells were incubated for another 30 minutes with a biotinylated anti-BrdU antibody (Sigma, Stockholm, Sweden). The immunoperoxidase procedure was carried out using Vectastain Elite ABC kit (ImmunKemi, Stockholm, Sweden), as described by the manufacturers. The diaminobenzidine tetrahydrochloridenickel (DAB-Ni) substrate kit was used as colour developer. Cells were analysed under fluorescence and visible light.

Another antibody used in the immunocytochemistry analysis was anti-c-Met (1:50) (Research Diagnostics). Freshly isolated progenitor cells (P0) and in vitro expanded cells in passages 6 (P6) and 12 (P12) were used for transplantation studies.

\section{Mice}

The animal care and use ethics committee at Huddinge Hospital approved the animal protocols. Acute liver injury was induced in C57 black/nude mice $(n=20)$, as described by Gupta et al, by administration of D-galactosamine (GalN) (Sigma Chemicals Co., Stockholm, Sweden) intraperitoneally at $0.7 \mathrm{~g} / \mathrm{kg}$ body weight. ${ }^{18}$ GalN was dissolved in PBS, pH 7.4, at $100 \mathrm{mg} / \mathrm{ml}$. Hepatic progenitor cells were transplanted into the spleen of 12 animals 36 hours after GalN treatment. Animals were anaesthetised under isoflurane, and $1 \times 10^{5}$ freshly isolated cells (P0) and $2 \times 10^{6}$ cells in passages 6 and 12 , suspended in $200 \mu \mathrm{l}$ of DMEM medium, were injected into the spleen over approximately $10-15$ seconds. Eight mice were sham transplanted with DMEM medium. After securing haemostasis, the abdominal incision was closed and animals were monitored closely until recovery.

Mice were killed four weeks after transplantation and the livers, spleens, and lungs were excised. Tissue pieces were shock frozen for immunohistochemical analysis. Cryosections, $5 \mu \mathrm{m}$ thickness, were air dried and fixed with cold 30\% acetone in methanol for 10 minutes and further analysed by immunohistochemistry.

\section{Immunohistochemistry}

For detection of human cells in mouse parenchyma, a mouse antihuman nuclei monoclonal antibody (Chemicon, California, USA), diluted 1:100, was used, followed by staining with biotinylated horse antimouse secondary antibody (1:500). ${ }^{19}$ The immunoperoxidase procedure was carried out using Vectastain Elite ABC kit (ImmunKemi, Stockholm, Sweden), as described by the manufacturers. The DAB-Ni substrate kit was used as colour developer. Other primary antibodies were non-conjugated antihuman albumin (Dakoppats, Denmark), antihuman CK19 (Neomarker), and antihuman CD26 (detects dipeptidyl peptidase IV) 
A

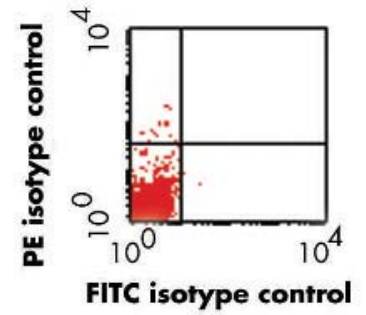

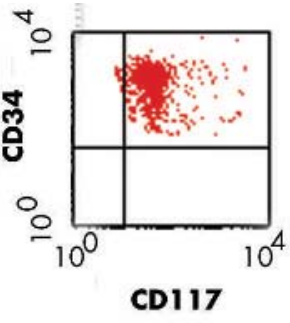

C
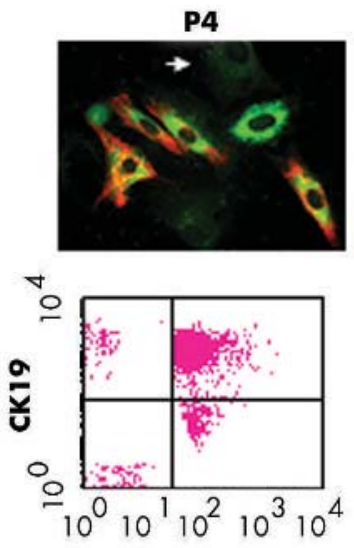

c-Met

D

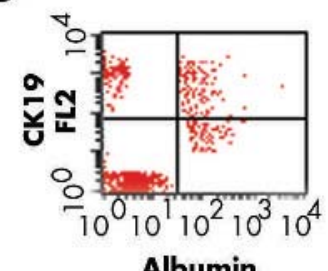

FL1

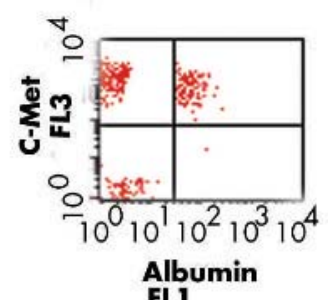

FLI

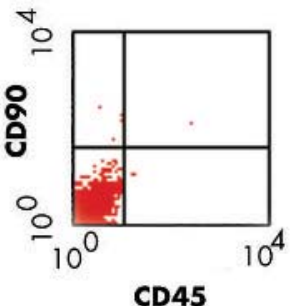

CD45

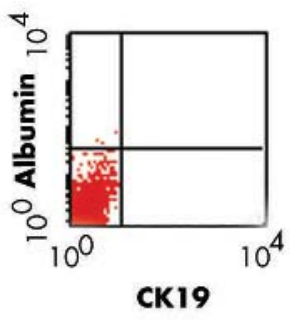

Albumin+/CK19+
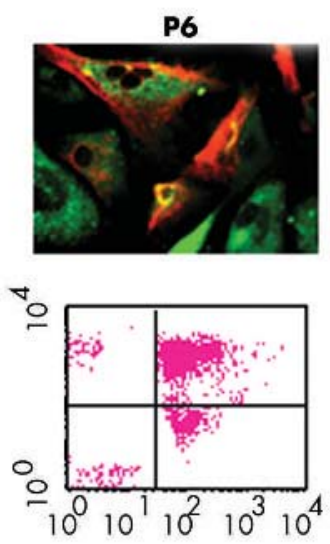

Albumin

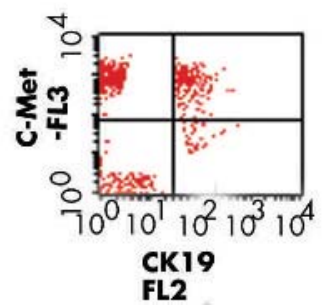

Figure 1 Characterisation of human hepatic progenitor cells. (A) Magnetically sorted fetal liver cells, immediately after isolation, stained positive for CD1 17 and CD34 but negative for CD90, CD45, albumin, and cytokeratin 19 (CK19). (B) CD117+/CD34+/Lin- cells, when cultured on collagen I coated culture dishes, grew in colonies. The first marker to be expressed on some cells was the hepatocyte growth factor receptor (c-Met, green). The nucleus is stained with DAPI (blue). Magnification $40 \times$. (C) Freshly isolated and expanded cells in various passages gave rise to albumin- CK19(white arrow), albumin+ (green) CK19+ (red), albumin+CK19- (green, hepatocytes), and albumin-CK19+ (red, cholangiocytes). Magnification 60×. (D) Three colour analysis demonstrated that c-Met was expressed on albumin+ and CK19+, but not albumin-/CK19- cells.

(Pharmingen) antibodies. Gamma glutamyl transpeptidase (GGT), glucose-6-phosphatase (G-6-P), and glycogen were demonstrated in situ, as described previously. ${ }^{20} 21$ Sections were counterstained with haematoxylin and mounted in mounting media (ImmunKemi).

We screened 60 serial sections of each mouse liver for DAB$\mathrm{Ni}$ positive human cells and determined the number of transplanted cells. A minimum of 100 high power fields in tissues from transplanted animal were analysed. The number of transplanted human hepatic progenitor cells per unit liver volume was determined as described previously. ${ }^{22}$

Reverse transcriptase-polymerase chain reaction (RT$P C R$ ) and real time RT-PCR

Total RNA was extracted from four human-mouse chimeric murine liver tissues and one sham transplanted mouse liver tissue, using the Micro-FastTrack RNA isolation kit (Invitrogen, Groningen, the Netherlands). Human specific primers were designed to detect human albumin, CK19, a fetoprotein, and $\alpha_{1}$ antitrypsin expression in mouse liver. Primers were selected using the Primer Express software version 2.0 (Applied Biosystems, Foster City, California,
USA). Each set of primers was designed to target cDNA alone, not contaminating DNA. Primer sets were commercially synthesised by CyberGene (Huddinge, Sweden).

Primer sequences were: CK19: sense, 5'-CCT GCG GGA CAA GAT TCT TG-3' and antisense, 5'-ACG GGC GTT GTC GAT CTG-3', expected product size (bp) 70; $\alpha$ fetoprotein: sense, 5'-GCA AAG CTG AAA ATG CAG TTG A-3' and antisense, 5'-GGA AAG TTC GGG TCC CAA AA-3', expected product size (bp) 129; albumin: sense, 5'-GCT TTG CCG AGG AGG GTA A-3' and antisense, 5'-GGT AGG CTG AGA TGC TTT TAA ATG T-3', expected product size (bp) 88; $\alpha_{1}$ antitrypsin: sense, 5'-CAG AGG AGG CAC CCC TGA A-3' and antisense, 5'-AGT CCC TTT CTC GTC GAT GGT-3', expected product size (bp) 71; and glucose-6-phosphate dehydrogenase (G-6-PD): sense, 5'-TGC CCC CGA CCG TCT AC-3' and antisense, 5' - ATG CGG TTC CAG CCT ATC TG-3', expected product size (bp) 76 .

Real time quantification of cDNA was performed using an ABI Prism 7000 Sequence Detection System (Applied Biosystems). To calculate relative gene expression, we used the comparative $\Delta \mathrm{Ct}$ method (User Bulletin \#2, ABI Prism 7700 SDS, Applied Biosystems). Results were calculated as 
A

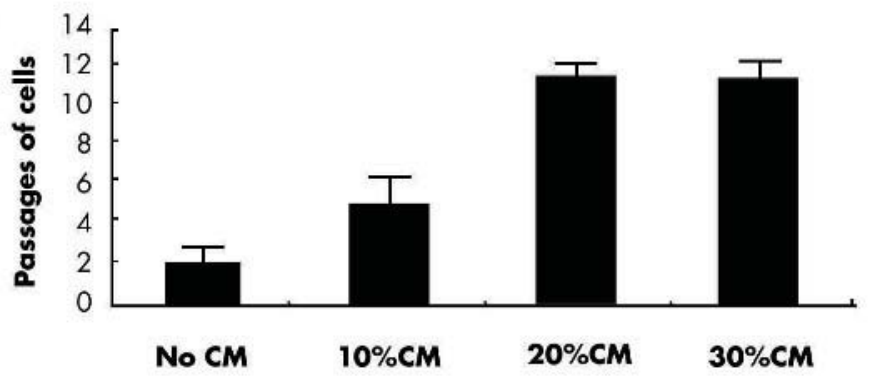

C

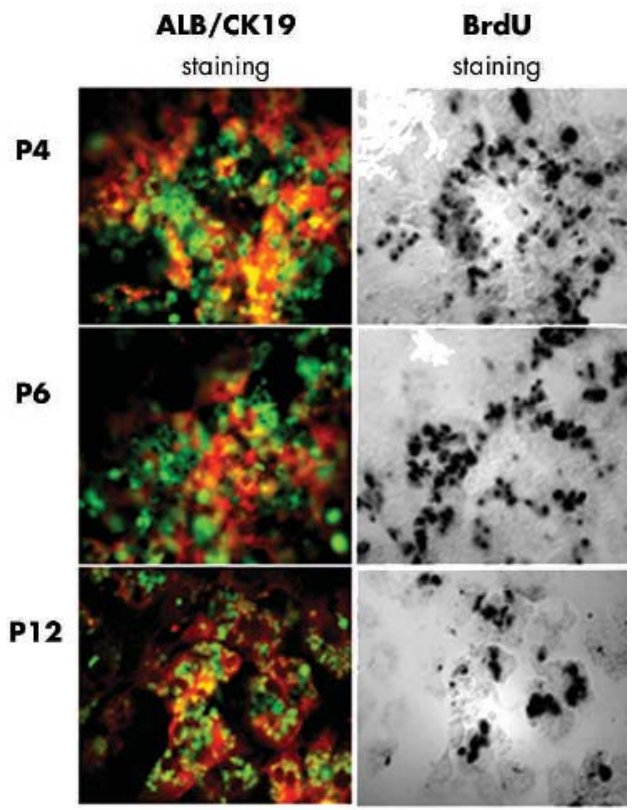

Phenotype

Figure 2 Proliferation of hepatic progenitors and their progeny. (A) Culture medium containing hepatocyte growth factor, epidermal growth factor, and basic fibroblast growth factor, when further supplemented with $20 \%$ conditioned medium (CM), significantly increased the proliferation of hepatic progenitor cells and could be passaged several times compared to without $C M(n=10 ; p<0.001$, Student's $t$ test). (B) Flow cytometric analysis revealed that high proliferation (BrdU+ cells) was observed in albumin+(ALB) cytokeratin (CK)19+, ALB+CK19-, and ALB-CK19+ cells, while double negative (ALB-CK19-) cells were more quiescent. (C) Liver progenitor cells in various passages, fluorescence stained for albumin (green) and CK19 (red) and enzymatically (diaminobenzidine tetrahydrochloride-nickel, black) for detection of BrdU incorporation, showing proliferative capacity in the various subpopulations. Magnification $40 \times$.

the ratio between the gene of interest (CK19, albumin, $\alpha$ fetoprotein, or $\alpha_{1}$ antitrypsin) and the endogenous control (G-6-PD) in the same sample. PCR reactions were performed as described previously. ${ }^{17}$

\section{Statistical methods}

The significance of differences was analysed using the Student's $t$ test. A p value of less than 0.05 was considered to be significant.

\begin{tabular}{|c|c|c|c|c|c|}
\hline $\begin{array}{l}\text { Embryonic } \\
\text { age of } \mathrm{FL} \\
\text { (weeks) }\end{array}$ & $\begin{array}{l}\text { Total No of } \\
\text { cells isolated } \\
\left(\times 10^{6}\right)\end{array}$ & $\begin{array}{l}\text { No of } \\
\text { CD117+/CD34+/Lin-cells } \\
\text { obtained }\left(\times 10^{4}\right)\end{array}$ & $\begin{array}{l}\text { Viability } \\
(\%)\end{array}$ & $\begin{array}{l}\text { No of cell } \\
\text { subpassages } \\
\text { obtained }\end{array}$ & $\begin{array}{l}\text { Approximate No } \\
\text { of cells obtained } \\
\text { at indicated } \\
\text { passage }\end{array}$ \\
\hline 6 & 6 & 3.1 & 93 & 4 & $\sim 5 \times 10^{5}$ \\
\hline 6 & 5.6 & 2 & 95 & 4 & $\sim 4.5 \times 10^{5}$ \\
\hline 6.5 & 10 & 4.5 & 94 & 2 & $\sim 8 \times 10^{5}$ \\
\hline 6.5 & 6.8 & 2.5 & 97 & 2 & $\sim 10 \times 10^{5}$ \\
\hline 8 & 8 & 3.2 & 92 & 2 & $\sim 7 \times 10^{4}$ \\
\hline 8.5 & 15 & 6.5 & 90 & 12 & $\sim 550 \times 10^{6}$ \\
\hline 8 & 7 & 3.2 & 95 & 12 & $\sim 400 \times 10^{6}$ \\
\hline 8.5 & 18 & 6.5 & 92 & 10 & $\sim 70 \times 10^{6}$ \\
\hline 9.5 & 13 & 5.5 & 97 & $9^{*}$ & $\sim 50 \times 10^{6}$ \\
\hline 9 & 11 & 7.5 & 94 & $12^{*}$ & $\sim 307 \times 10^{6}$ \\
\hline 9 & 5.6 & 4.5 & 93 & 12 & $\sim 360 \times 10^{6}$ \\
\hline 9.5 & 10.5 & 7.5 & 92 & $12^{*}$ & $\sim 320 \times 10^{6}$ \\
\hline 9.5 & 17.6 & 8.8 & 95 & $12^{*}$ & $\sim 360 \times 10^{6}$ \\
\hline 9 & 14 & 13.3 & 96 & 11 & $\sim 272 \times 10^{6}$ \\
\hline 9.5 & 15.5 & 9.5 & 97 & $14^{*}$ & $\sim 389 \times 10^{6}$ \\
\hline 9.5 & 13.6 & 12 & 91 & 11 & $\sim 245 \times 10^{6}$ \\
\hline
\end{tabular}

${ }^{*}$ Cells still in culture at present

$\mathrm{FL}$, fetal liver. 


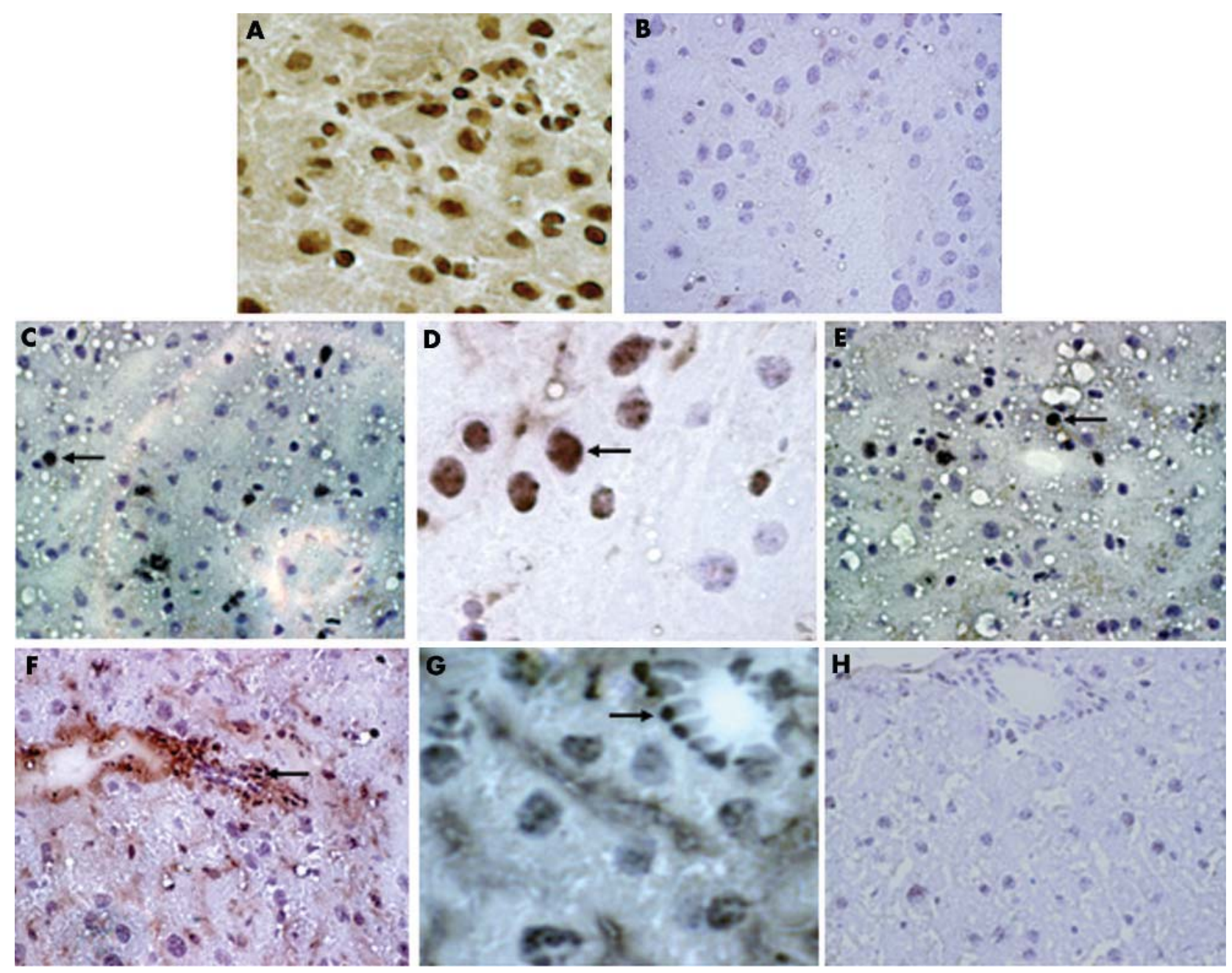

Figure 3 Detection of human hepatic progenitor cells in mouse liver. (A) The antihuman nuclei antibody stained normal human liver cells (brown/ black), but not cells of the sham transplanted mouse (B), demonstrating the specificity of the antibody. (C) Freshly isolated CD1 17+/CD34+/Lin-, (D) sixth, and (E) 12th passage human hepatic progenitors cells when transplanted into D-galactosamine treated mice showed differentiation into hepatocytes. Haematoxylin counterstain was used to distinguish mouse cells (blue/grey) from human cells (brown/black). (F, G) Human cells also repopulated areas of mouse bile ducts. (H) A sham transplanted control mouse bile duct. Magnification $60 \times$.

\section{RESULTS \\ CD $117+/$ CD34+/Lin - liver progenitor cells can differentiate into hepatocytes and cholangiocytes in vitro}

Using a kit designed to isolate primitive haematopoietic progenitors, we obtained a population of cells from human FLs (gestational weeks 6-9) that did not express any committed haematopoietic markers. Further phenotyping of this population showed expression of the stem cell markers CD117 and CD34 but no expression of liver markers such as albumin (hepatocyte marker) and CK19 (cholangiocyte marker). Nor was there any expression of Thy-1 (CD90) or CD45 (fig 1A). This population represents approximately 0.5 $0.7 \%$ of whole FLs for gestational weeks 6-9. Expression of CD45 and CD90 was not observed during subculture of the cells. In our hands, the isolation procedure gave $>98 \%$ pure populations of CD117+/CD34+/Lin- cells, as determined by the FACS analysis. On cultivation, we found these cells to be a mixture of both adherent ( $85 \%$ ) (fig $1 \mathrm{~B}$ ) and nonadherent populations $(\sim 15 \%)$. The non-adherent population could not be expanded further under the culture conditions given below. CD117+/CD34+/Lin - cells and their progeny tended to grow in colonies (fig 1B).
We found that the first marker to be expressed by some of the adherent progenitor cells after two days in culture was c-Met (fig 1B). In vitro cultivation of these cells for two weeks in culture medium containing HGF and EGF showed the presence of four types of cells: (i) $\sim 85 \%$ double positive cells expressing albumin and CK19, (ii) 4\% double negative cells, (iii) $\sim 6 \%$ single positive cells expressing only albumin, and (iv) $\sim 5 \%$ single positive cells expressing only CK19 (fig lC). This phenotype was maintained for several passages during cultivation (fig 1C). However, from P1l onwards there was a slight decrease in the numbers of double positive $(\sim 60 \%)$ and double negative $(\sim 3 \%)$ cells while single positive cells for albumin or CK19 increased. Three colour analysis showed that cells expressing c-Met were albumin+ and CK19+ but not albumin-CK19- (fig 1D).

\section{Liver progenitor cells can be passaged extensively in vitro}

Proliferation of liver progenitors was dependent on the regular use of $20 \% \mathrm{CM}$ during cultivation. Using 20\% CM, we can passage these cells to at least 12 passages. Removal of $\mathrm{CM}$ significantly decreased the number of cell passages obtained $(\mathrm{p}<0.001$, fig $2 \mathrm{~A})$, reflecting a decrease in the 


\section{Human hepatic progenitor transplanted}
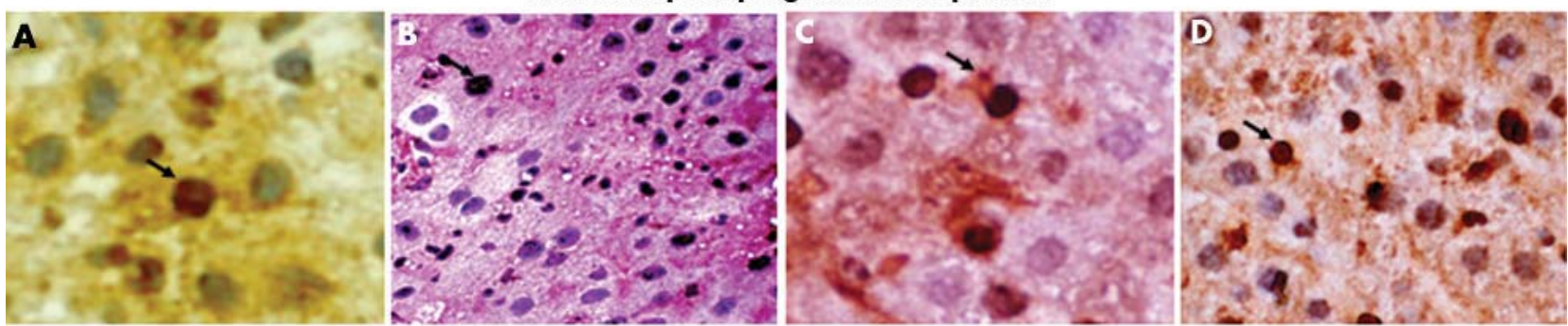

\section{Sham transplanted}
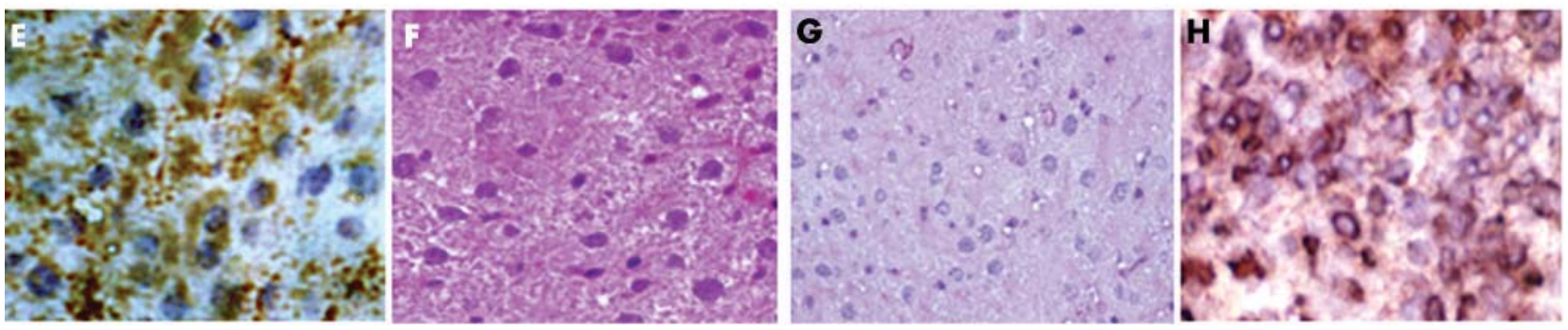

Figure 4 In vivo fate of human hepatic progenitor cells. Human hepatic progenitor cells one month after transplantation in D-galactosamine treated mice contained (A) glucose-6-phosphatase (G-6-P, brown cytoplasmic staining), (B) glycogen (pink), (C) dipeptidyl peptidase IV (red-brown), and (D) gamma glutamyl transpeptidase (GGT, red/brown cytoplasmic staining). (E-H) Staining pattern for the same markers in sham transplanted (receiving only culture medium) control mice are shown. Note that G-6-P, glycogen, and GGT are non-human specific markers and are therefore also detected in sham transplanted mice. Tissue sections were counterstained with haematoxylin which stains mouse cells (blue). Magnification $60 \times$.

proliferation of cells. We found that a high proliferative capacity was observed in double positive cells (albumin+CK19+) and the single albumin positive cells (albumin+CK19-), as detected by incorporation of BrdU (fig 2B, C). Cells can be maintained for long periods with stable phenotype. It is important to note that not all FLs generated cells which proliferated rapidly and which could be maintained in culture for several passages (table 1). Successful proliferation for long periods was dependent on the quality of FL tissue obtained. However, we found that CD117+/CD34+/Lin - cells isolated from all FLs tested differentiated into hepatic cells. Those cells that stopped
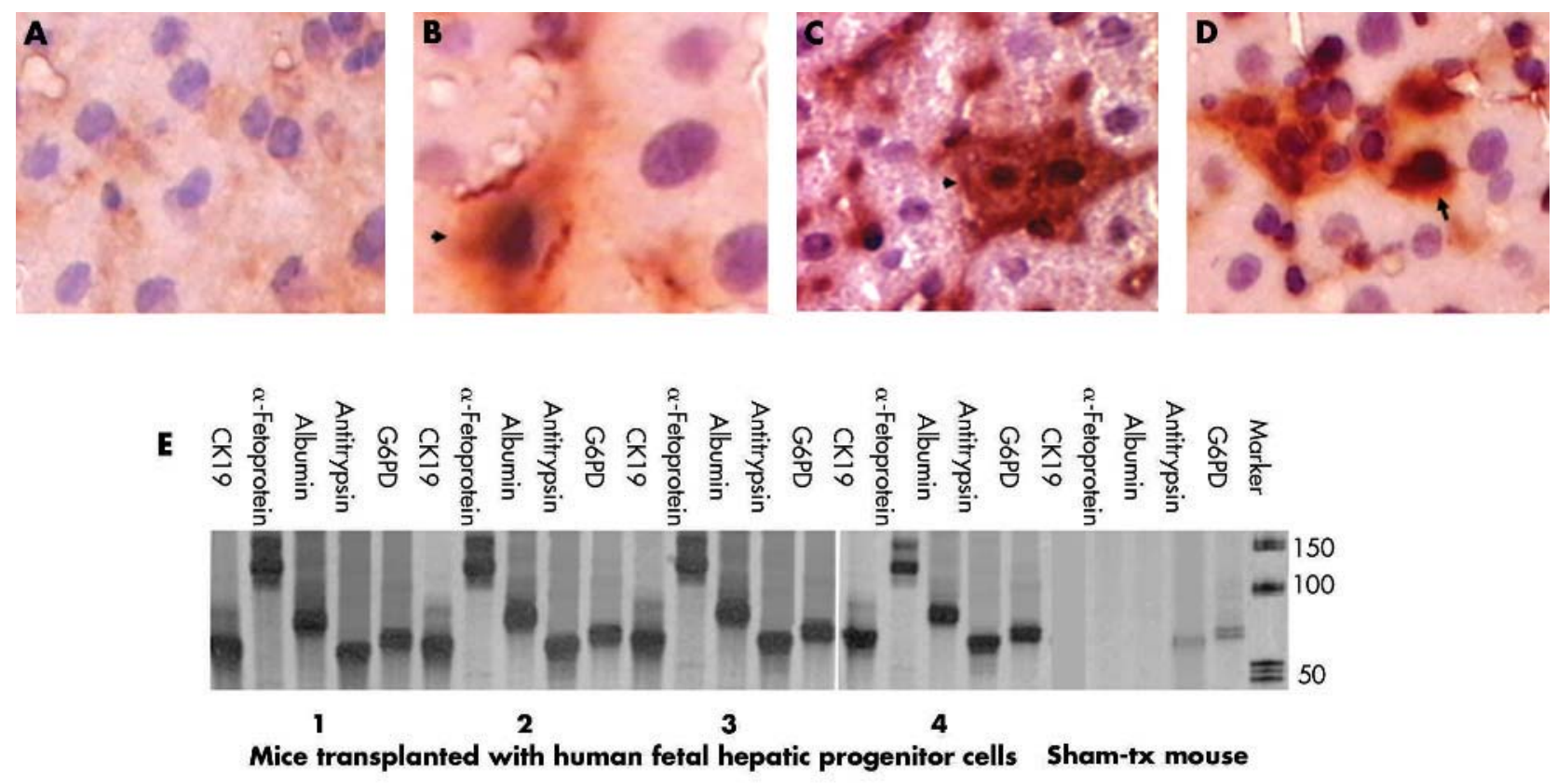

Figure 5 In vivo fate of human hepatic progenitor cells. (A) Staining of sham transplanted mouse liver with the antihuman albumin antibody (red). Differentiation of (B) freshly isolated CD1 17+/CD34+/Lin-, (C) sixth, and (D) 12th passage human hepatic progenitors cells into mature hepatocytes The transplanted human cells double stained for albumin (red) and human nuclei (diaminobenzidine tetrahydrochloride-nickel, black (arrowheads)). All sections were counterstained with haematoxylin to distinguish mouse cells (blue) from human cells (brown/black). Magnification 60x. (E) Transcription of human liver specific genes in the mouse liver. Human cytokeratin 19 (CK19), $\alpha$-fetoprotein, and albumin were detected in the livers of D-galactosamine treated mice that received human hepatic progenitor cells but not in sham transplanted mice. However, $\alpha_{1}$ antitrypsin was also slightly amplified in controls. Glucose-6-phosphate dehydrogenase (G6PD) was used as the housekeeping gene. 
Table 2 Human liver progenitor cell engraftment in Dgalactosamine treated mice at four weeks after transplantation

\begin{tabular}{llll}
\hline $\begin{array}{l}\text { Cell } \\
\text { passage } \\
\text { No }\end{array}$ & $\begin{array}{l}\text { No of cells } \\
\text { transplanted }\end{array}$ & $\begin{array}{l}\text { Transplanted } \\
\text { human cells } \\
\text { detected } / \mathrm{cm}^{3} \\
\times 10^{6}\end{array}$ & $\begin{array}{l}\text { Approximate \% of host } \\
\text { hepatocyte mass } \\
\text { repopulated by } \\
\text { human cells }\end{array}$ \\
\hline P0 & $1 \times 10^{5}$ & $3.0(0.2)$ & 4 \\
P0 & $1 \times 10^{6}$ & $4.8(1.6)$ & 5 \\
P6 & & & \\
P6 & & & \\
P6 & & $5.1(2.2)$ & 5 \\
P6 & $1 \times 10^{6}$ & & \\
P12 & & & \\
P12 & & & \\
P12 & & & \\
P12 & & & \\
\hline
\end{tabular}

proliferating after a certain number of passages were polyploid and became senescent.

Expanded human fetal progenitor cells successfully differentiate into functionally mature hepatocytes and cholangiocytes in the livers of GaIN treated mice

To test whether freshly isolated CD117+/CD34+/Lin- cells (P0) and cells expanded in culture (P6 and P12) have the potential to differentiate and be functional in vivo, we transplanted these cells into mice that were treated with GalN to induce acute liver injury. The mortality rate of GalN treated mice in the control group ( sham transplanted, $\mathrm{n}=8$ ) was $62 \%(\mathrm{n}=5)$ and in the test group (human cell transplanted, $\mathrm{n}=12)$ was $25 \%(\mathrm{n}=3)$ at $24-72$ hours after treatment $(\mathrm{p}<0.01)$. No increased mortality was observed in either groups after 72 hours.

The species specificity of the antihuman nuclei antibody is demonstrated by the positive result with human liver (fig 3A) and the negative immunohistochemistry with sham transplanted livers from nude mice (fig 3B). Furthermore, no human cells were observed in the spleen and lungs of transplanted mice (data not shown).

Freshly isolated CD117+/CD34+/Lin- cells (P0) and hepatic progenitor cells in P6 and P12, when transplanted, differentiated into hepatocytes (fig 3C-E) four weeks after transplantation. Areas of bile ducts repopulated by human progenitors were observed in animals transplanted with human cells but not in sham transplanted animals (fig 3F$\mathrm{H})$. Human transplanted cells expressed hepatocyte markers such as G-6-P (fig 4A) and glycogen (fig 4B), and biliary markers such as dipeptidyl peptidase IV (fig 4C) and GGT (fig 4D). The same markers expressed in control sham transplanted mice are shown in fig 4E-H. A monoclonal antibody against human albumin was used to examine expression of human albumin in transplanted cells. Negative immunohistochemistry with sham transplanted livers from nude mice (fig 5A) showed the species specificity of the antibody. We found several albumin positive human transplanted cells (fig 5B-D) in all transplanted mice. Of note, no tumour formations were observed in any of the mice analysed.

We estimated that approximately $4-5 \%$ of the host hepatocyte mass was repopulated by the transplanted human hepatic progenitor cells (table 2 ). Furthermore, there was no significant difference in the number of engrafted transplanted cells between animals receiving P0, P6, and P12 cells (table 2).

Transcription of human liver specific genes in mice transplanted with human hepatic progenitor cells We further confirmed engraftment of transplanted cells by determining expression of human genes in transplanted mice. We analysed livers of mice sacrificed one month after transplantation of human fetal hepatic progenitor cells by RT-PCR using primers specific for human liver specific genes, including albumin, $\alpha_{1}$ antitrypsin, CK19, and $\alpha$ fetoprotein. RNA from the liver of a sham transplanted nude mouse resulted in amplification for G-6-PD which was used as a control for the integrity of the RNA. CK19, albumin, and $\alpha$ fetoprotein primers were species specific for human genes as they did not amplify the respective mouse genes but $\alpha_{1}$ antitrypsin was not found to be species specific (fig $5 \mathrm{E}$ ). Quantitative analysis of the genes for human CK19, $\alpha$ fetoprotein, albumin, and antitrypsin in four mice receiving human cells is shown in table 3.

\section{DISCUSSION}

The main aim of the present study was identification of a specific population of hepatic progenitor cells with extensive proliferation capacity in vitro and, when transplanted in vivo, could differentiate into mature hepatic cells. Our results showed that CD117+/CD34+/Lin- cells belong to a population of human liver progenitors that can be expanded extensively in vitro. On transplantation, this population can differentiate into functional hepatic cells in an acute liver injury model. Engraftment studies showed that approximately $5 \%$ of the host hepatocyte mass was repopulated by human hepatic progenitor cells, which is similar to other reports using hepatocyte cell transplantation. ${ }^{22}{ }^{23}$

Prior to transplantation, freshly isolated liver progenitors did not express albumin or CK19. However, after transplantation, these cells differentiated into mature hepatocytes and cholangiocytes, as determined by expression of mature hepatic markers. These cells expressed the two haematopoietic associated markers c-kit and CD34, but not CD45, the marker that distinguishes haematopoietic cells from nonhaematopoietic cells. Further assays to determine the exact origin of these cells are required.

CD117+/CD34+/Lin - cells in culture gave rise to four types of cells: albumin-CK19-, albumin-CK19+, albumin+ CK19- hepatocytes, and albumin-CK19+ cholangiocytes. We currently do not know which factors in the CM favour the

Table 3 Quantitative analysis of human liver specific genes expressed in D-galactosamine treated mice transplanted with human liver progenitor cells

\begin{tabular}{lccccc}
\hline & \multicolumn{6}{c}{ Gene expression compared with G-6-PD (\%) } \\
\cline { 2 - 6 } Human specific gene & Mouse 1 & Mouse 2 & Mouse 3 & Mouse 4 & Sham tx mouse \\
\hline Passage of transplanted cells & P0 & P6 & P6 & P12 & Only medium \\
CK19 & 186 & 243 & 155 & 114 & 0 \\
$\alpha$-Fetoprotein & 7 & 3 & 4 & 1 & 0 \\
Albumin & 47 & 58 & 60 & 65 & 0 \\
$\alpha_{1}$ Antitrypsin & 3256 & 2003 & 2425 & 1945 & 168 \\
\hline
\end{tabular}

G-6-PD, glucose-6-phosphate dehydrogenase; CK, cyłokeratin. 
growth of liver progenitor cells. Except for the double negative cells, all other subpopulations had a high fraction of proliferating cells (BrdU+). The albumin-CK19- cell subset were quiescent in vitro; nevertheless, this cell population may be responsible for maintaining the pool of expanding cells and may thus represent the population of true hepatic stem cells. In some cases CD117+/CD34+/Lincells and their progeny could be passaged for a maximum of 12 passages. At this stage many of the differentiated maturing hepatocytes exhibited increasing polyploidy, which is known to attenuate cell proliferation and eventually produces cell senescence. ${ }^{24-26}$ On the other hand, at present we have hepatic progenitor cells from five different liver progenitor cell lines in culture which have been passaged with a stable progenitor phenotype more than 12 times. We are currently testing telomerase activity in these cells as a possible explanation for their extensive replication. It is important to note that only cells from FLs from gestational week 8.5 onwards could be successfully expanded to large numbers. Despite the high proliferative capacity of these cells, no tumours were observed in vivo four weeks after transplantation.

One of the aims was to determine whether in vitro expanded human hepatic progenitors could differentiate into mature hepatic cells in vivo. For this purpose we used the GalN induced acute liver injury model described previously. ${ }^{18}{ }^{27}$ GalN treatment is known to cause characteristic liver damage resulting in panlobular liver injury with extensive macro and microvesicular fatty changes. This is accompanied by extensive hydropic changes in hepatocytes, as well as areas of cell drop out. Other changes caused by GalN have been well described elsewhere. ${ }^{18} 27$ We were able to confirm these changes in our mice treated with GalN (data not shown). The mortality rate in GalN treated animals receiving human cells (irrespective of cell passage) was significantly lower than sham treated mice, indicating a probable therapeutic effect of human transplanted cells. It has been reported that when cells are transplanted during GalN induced acute liver injury, engraftment of the transplanted cells is not disrupted. ${ }^{28}{ }^{29}$ Thus D-GalN induced injury may enable successful engraftment and differentiation of human cells and may in part explain the therapeutic effect of transplanted cells. However, the therapeutic effect of these cells needs to be confirmed in a larger study. In addition, further investigations to study details of engraftment and in vivo proliferation of these cells are required. These are important issues that need to be addressed in order to make transplantation with FL progenitors a reality in the future. Such studies are currently underway at our centre. In addition, further studies to determine whether liver engraftment is comparable between human hepatic progenitors in various passages are ongoing.

Ex vivo expanded cells have tremendous potential applications, not only for transplantation purposes but also to test candidate therapeutic drugs for liver toxicity and metabolism. Hepatitis $\mathrm{C}$ virus (HCV) infection is a worldwide problem and currently there are no vaccines or drugs that specifically target hepatitis $\mathrm{C}$ virus. ${ }^{30}$ The lack of well defined in vitro and in vivo models of HCV infection has slowed critical research that could help identify more effective therapies. Fetal liver cells differentiated into hepatocytes in vitro can be used to study the molecular mechanisms of HCV replication and to screen drugs that target this process which may prove to be effective HCV therapies in the future.

\section{ACKNOWLEDGEMENTS}

The present study was financed by grants from the Medical Research Council No K2002-06X-14004-02B, the Magnus Bergvall, the Karolinska University Hospital Research Council and the Lars Erik Gelins Foundations to Dr Sumitran-Holgersson.

\section{Authors' affiliations}

G Nowak, B-G Ericzon, S Nava, S Sumitran-Holgersson, Division of

Transplantation Surgery, Karolinska University Hospital-Huddinge, Karolinska Institutet, Stockholm, Sweden

M Jaksch, Division of Clinical Immunology, Karolinska University Hospital-Huddinge, Karolinska Institutet, Stockholm, Sweden

M Westgren, Division of Obstetrics and Gynaecology, Karolinska

University Hospital-Huddinge, Karolinska Institutet, Stockholm, Sweden

Conflict of interest: None declared.

\section{REFERENCES}

1 Van Thiel DH, Makowka L, Starzl TE. Liver transplantation: where it's been and where it's going. Gastroenterol Clin North Am 1988;17:1-18.

2 Gupta S, Gorla GR, Irani AN. Hepatocytic transplantation: emerging insights into mechanisms of liver repopulation and their relevance to potential therapies. J Hepatol 1999;30:162-70.

3 Rhim JA, Sandgren EP, Degen JL, et al. Replacement of diseased mouse liver by hepatic cell transplantation. Science 1994;263:1149-52.

4 Overturf K, Al-Dhalimy M, Tanguay R, et al. Hepatocytes corrected by gene therapy are selected in vivo in a murine model of hereditary tyrosinemia type 1. Nat Genet 1996;12:266-73.

5 Theise ND. Liver stem cells: prospects for treatment of inherited and acquired liver diseases. Expert Opin Biol Ther 2003;3:403-8.

6 Lagasse E, Connors H, Al-Dhalimy M, et al. Purified hematopoietic stem cells can differentiate into hepatocytes in vivo. Nat Med 2000;6:1229-34

7 Korbling M, Katz RL, Khanna A, et al. Hepatocytes and epithelial cells of donor origin in recipients of peripheral-blood stem cells. NEngl J Med 2002;346:738-46. 8 Alison MR, Poulsom R, Jeffery R, et al. Hepatocytes from non-hepatic adult stem cells. Nature 2000:406:257.

9 Suzuki A, Zheng YW, Kaneko S, et al. Clonal identification and characterization of self-renewing pluripotent stem cells in the developing liver. J Cell Biol 2002;156:173-84.

10 Harmeet M, Irani AN, Gagandeep S, et al. Isolation of human progenitor liver epithelial cells with extensive replication capacity and differentiation into mature hepatocytes. J Cell Sci 2002;115:2679-88.

11 Wang X, Ge S, McNamara G, et al. Albumin-expressing hepatocyte-like cells develop in the livers of immune-deficient mice that received transplants of highly purified human hematopoietic stem cells. Blood 2003;101:4201-8.

12 Petersen BE, Goff JP, Greenberger JS, et al. Hepatic oval cells express the haematopoietic stem cell marker Thy-1 in the rat. Hepatology 1998;27:433-45.

13 Crosby HA, Kelly DA, Strain AJ. Human hepatic stem-like cells isolated using c-kit or CD34 can differentiate into biliary epithelium. Gastroenterology $2001 ; 120: 534-44$

14 Muench MO, Cupp J, Polakoff J, et al. Expression of CD33, CD38, and HLA$\mathrm{DR}$ on CD34+ human fetal liver progenitors with a high proliferative potential. Blood 1994;83:3170-81.

15 Westgren M, Ek S, Bui TH, et al. Establishment of a tissue bank for fetal stem cell transplantation. Acta Obstet Gynecol Scand 1994;73:385-8.

16 England MA. A color atlas of life before birth. Normal fetal development. London: Wolfe Medical Publications, 1988.

17 Bo X, Broomé U, Uzunel M, et al. Capillarization of hepatic sinusoid by liver endothelial cell-reactive autoantibodies in patients with cirrhosis and chronic hepatitis. Am J Pathol 2003;163:1275-89.

18 Gupta S, Rajvanshi $P$, Irani AD, et al. Integration and proliferation of transplanted cells in hepatic parenchyma following D-galactoseamineinduced acute injury in F344 rats. J Pathol 2000;190:203-10.

19 Kohama I, Lankford KL, Preiningerov J, et al. Transplantation of cryopreserved adult human schwann cells enhances axonal conduction in demyelinated spinal cord. J Neuroscience $2001 ; 21: 944-50$.

20 Rutenberg AM. Histochemical and ultrastructural demonstration of $\gamma$-glutamyl transpeptidase activity. J Histochem Cytochem 1969;17:517-26.

21 Ott M, Rajvanshi P, Sokhi RP, et al. Differentiation-specific regulation of transgene expression in a diploid epithelial cell line derived from the normal F344 rat liver. J Pathol 1999;187:365-73.

22 Rajvanshi P, Kerr A, Kuldeep KB, et al. Efficacy and safety of repeated hepatocyte transplantation for significant liver repopulation in rodents. Gastroenterology 1996;111:1092-102.

23 Ponder KP, Gupta S, Leland F, et al. Mouse hepatocytes migrate to liver parenchyma and function indefinitely after intrasplenic transplantation. Proc Natl Acad Sci U S A 1991;88:1217-21.

24 Sigal S, Gupta S, Gebhardt DF Jr, et al. Evidence for a terminal differentiation process in the liver. Differentiation 1995;59:35-42.

25 Sigal S, Rajvanshi P, Gorla GR, et al. Partial hepatectomy-induced polyploid attenuates hepatocyte replication and activates cell aging events. Am J Physiol 1999;276:G1260-72

26 Gorla GR, Malhi H, Gupta S. Polyploidy associated with oxidative DNA injury attenuates proliferative potential of cells. J Cell Sci 2001;114:2943-51.

27 Dabeva MD, Shafritz DA. Activation, proliferaion, and differentiation of progenitor cells into hepatocytes in the D-galactosamine model of liver regeneration. Am J Pathol 1993;143:1606-20.

28 Rajvanshi P, Gupta S. Rapid integration and proliferation of transplanted hepatocytes in D-galactosamine-injured rat liver indicates potential of cell transplantation in acute liver failure. Hepatology 1995;22:213A.

29 Gupta S, Rajvanshi P, Sokhi RP, et al. Mechanisms determining augmentation of the transplanted hepatocyte mass with growth stimulating signals. Hepatology 1997;26:167A.

30 Burroughs A, McNamara D. Liver diseases in europe. Aliment Pharmacol Ther 2003; 18:54-9. 